\title{
Rolipram stimulates angiogenesis and attenuates neuronal apoptosis through the cAMP/cAMP-responsive element binding protein pathway following ischemic stroke in rats
}

\author{
SHOUYE HU ${ }^{1,2 *}$, QINGWEN CAO ${ }^{2 *}$, PENG XU ${ }^{1,2}$, WENCHEN $\mathrm{JI}^{2}$, GANG WANG $^{2,3}$ and YUELIN ZHANG ${ }^{2,3}$ \\ ${ }^{1}$ Department of Osteonecrosis and Joint Reconstruction, Xi'an Honghui Hospital; \\ ${ }^{2}$ Xi'an Jiaotong University Health Science Center; ${ }^{3}$ Department of Neurosurgery, \\ Shaanxi Province People's Hospital, Xi'an, Shaanxi 710068, P.R. China \\ Received February 4, 2014; Accepted June 13, 2014
}

DOI: $10.3892 /$ etm.2015.2958

\begin{abstract}
Rolipram, a phosphodiesterase-4 inhibitor, can activate the cyclic adenosine monophosphate (cAMP)/cAMP-responsive element binding protein (CREB) pathway to facilitate functional recovery following ischemic stroke. However, to date, the effects of rolipram on angiogenesis and cerebral ischemia-induced neuronal apoptosis are yet to be fully elucidated. In this study, the aim was to reveal the effect of rolipram on the angiogenesis and neuronal apoptosis following brain cerebral ischemia. Rat models of ischemic stroke were established following transient middle cerebral artery occlusion and rolipram was administered for three, seven and 14 days. The results were examined using behavioral tests, triphenyl tetrazolium chloride staining, immunostaining and terminal deoxynucleotidyl transferase-mediated dUTP nick end labeling (TUNEL) to evaluate the effects of rolipram therapy on functional outcome, angiogenesis and apoptosis. Western blot analysis was used to show the phosphorylated- (p-)CREB protein level in the ischemic hemisphere. The rolipram treatment group exhibited a marked reduction in infarct size and modified neurological severity score compared with the vehicle group, and rolipram treatment significantly promoted the microvessel density in the ischemic boundary region and increased p-CREB protein levels in the ischemic hemisphere. Furthermore, a significant reduction in the number of TUNEL-positive cells was observed in the rolipram group compared with the vehicle group. These findings suggest that rolipram has the ability to
\end{abstract}

Correspondence to: Professor Peng Xu, Department of Osteonecrosis and Joint Reconstruction, Xi'an Honghui Hospital, 555 Xi'an Youyi East Road, Xi'an, Shaanxi 710068, P.R. China E-mail: sousou369@163.com

*Contributed equally

Key words: rolipram, angiogenesis, neuronal apoptosis, cAMP/cAMP-responsive element binding protein pathway, rat attenuate cerebral ischemic injury, stimulate angiogenesis and reduce neuronal apoptosis though the cAMP/CREB pathway.

\section{Introduction}

Ischemic stroke is induced by cerebral artery occlusion, which can cause regional cerebral flow reduction or interruption (1). The brain is sensitive to ischemia, the effect of which may be exacerbated by the reperfusion. Necrotic cells begin to die within a few minutes of injury. Furthermore, the neuronal cells in the cerebral cortex, striatum and hippocampus begin to die within several hours after ischemic stroke and the process of cell death may last several days (2). Although thrombolytic therapy is considered to be the only beneficial treatment in clinical practice, the majority of patients with ischemic stroke still fail to receive adequate treatment in time $(3,4)$. Previous studies have revealed that enhancing angiogenesis and reducing the apoptosis of nerve cells may improve clinical outcomes during the recovery phase following an ischemic stroke. Furthermore, increasing evidence has indicated that the cyclic adenosine monophosphate (cAMP)-responsive element binding protein (CREB) signaling pathway is intimately involved in a variety of nerve protection mechanisms following ischemic stroke (5), and that the phosphorylation of CREB plays a critical role in learning and memory function $(6,7)$. Phosphorylation of CREB can be achieved by a number of upstream signaling cascades, including the cAMP-protein kinase A (PKA) cascade $(8,9)$, and the $\mathrm{CAMP} / \mathrm{CREB}$ pathway exerts a strong effect on the development, survival, maturation and integration of new neurons $(10,11)$. This has prompted the theory that the cAMP/CREB pathway may provide benefits for brain remodeling following ischemic injury and may be a target of cerebral ischemia treatment. However, a limited number of studies have investigated whether the $\mathrm{AMP} / \mathrm{CREB}$ pathway is involved in the process of angiogenesis and apoptosis following cerebral ischemia/reperfusion injury $(12,13)$.

Rolipram typically acts as an antidepressant- and anxiolytic-like agent (14); however, a number of studies have revealed that it may reduce the infarction area caused by cerebral ischemia (15) and also increase the phosphorylated- (p-) CREB expression level in the hippocampus $(14,16)$. This study 
focused on the protective effect of rolipram on transient cerebral ischemia/reperfusion injury in rats, and aimed to investigate the hypothesis that rolipram acts through promoting angiogenesis and reducing apoptosis following cerebral ischemia.

\section{Materials and methods}

Experimental animals. Male Wistar rats, weighing 250-300 g, were obtained from the Center of Experimental Animals, School of Medicine (Xi'an Jiaotong University, Xi'an, China). The rats were maintained on a 12-h light/dark cycle and allowed free access to food and water. All the experiments were approved and supervised by the Animal Care Committee of Xi'an Jiaotong University Health Science Center.

Transient middle cerebral artery occlusion (tMCAO). Prior to the surgery, the rats were fasted overnight but allowed free access to water. In brief, the rats were anesthetized using chloral hydrate $[350 \mathrm{mg} / \mathrm{kg}$, intraperitoneal (i.p.)]. The rectal temperature was monitored and maintained at $37.0 \pm 0.5^{\circ} \mathrm{C}$, using a feedback-regulated heating system during the surgery. tMCAO (17) was induced by the method of intraluminal vascular occlusion. Briefly, a 4-0-nylon monofilament suture with a slightly enlarged round tip was inserted into the stump of the external carotid artery (ECA) and run across the lumen of the internal carotid artery, until it reached and occluded the MCA. The average distance between the bifurcation of the common carotid artery and the tip of the suture inserted to occlude the MCA was 18-20 mm. Two hours after MCAO, reperfusion was achieved with the withdrawal of the suture until the tip cleared the lumen of the ECA. Sham-operated animals were subjected to the above-described procedures, with the exception of suture insertion.

Rolipram treatment. Ischemic rats received injections of rolipram (3 mg/kg, vehicle i.p.; Sigma-Aldrich, St. Louis, MO, USA) from the first day after ischemia. The treatment lasted three, seven and 14 consecutive days according to the group. The dosage and dosing frequency of rolipram were selected on the basis of previous studies (18). The rats were randomly divided into five groups according to the tMCAO insult, sham procedure and drug use: i) Sham group, rats underwent the surgical procedure but without tMCAO; ii) vehicle group, rats underwent tMCAO and received $0.9 \%$ saline treatments; iii) three days group, rats underwent $\mathrm{tMCAO}$ and received a three-day rolipram course; iv) seven days group, rats underwent tMCAO and received a seven-day rolipram course; v) 14 days group, rats underwent tMCAO and received a 14-day rolipram course. Food and water were freely accessible throughout the experimental course.

Assessing cerebral infarction and functional outcome. The functional outcome in the rats was evaluated by the modified neurological severity score (mNSS), for which the rats were assessed using several tests, including raising the rat by the tail, placing the rat on the floor and beam balance walking. All the test scores were incorporated into the mNSS (1). In addition, triphenyl tetrazolium chloride (TTC) staining (1) was used to evaluate the brain infarction size. The colorless TTC is reduced to a red formazan produced by dehydrogenases, which are most abundant in mitochondria. As such, TTC staining is a functional test of dehydrogenase enzyme activity and is usually used for the early histochemical diagnosis of infarction. Therefore, the rats were sacrificed at three days post-surgery to assess the infarction change using TTC. Subsequent to documenting the mNSS for 21 days, the rats were all sacrificed and immunochemical staining was performed. The brain tissue was rapidly removed, immersed in cold saline for $10 \mathrm{~min}$ and sliced into $2.0 \mathrm{~mm}$-sections. The brain slices were incubated in $2 \%$ TTC dissolved in phosphate-buffered saline for $30 \mathrm{~min}$ at $37^{\circ} \mathrm{C}$ and then transferred to a $4 \%$ formaldehyde solution for fixation.

Western blot analysis. p-CREB levels were estimated by western blot analysis, and $\beta$-actin was utilized as a loading control. The animals were sacrificed at three, seven and 14 days after surgery. The right ischemic hemispheres were collected. Protein samples were homogenized in radioimmunoprecipitation assay buffer. A total of $60 \mu \mathrm{g}$ protein was loaded in each lane, and the proteins were separated by $10 \%$ SDS-PAGE and electroblotted onto nitrocellulose membranes (Millipore, Billerica, MA, USA). Subsequent to blockage with 5\% non-fat milk, the blots were incubated with rabbit anti-p-CREB (1:400; Santa Cruz Biotechnology, Inc., Santa Cruz, CA, USA) and $\beta$-actin conjugated goat anti-rabbit (1:5,000; Santa Cruz Biotechnology, Inc.) primary antibodies. $\beta$-actin was used as an internal reference for relative quantification.

Immunohistochemistry. Animals were maintained for 20 days after MCAO and then sacrificed with chloral hydrate (400 mg/kg, i.p.). The rat brains were fixed by transcardial perfusion with saline, followed by perfusion and immersion in $4 \%$ paraformaldehyde. Using a microtome, serial coronal sections (4- $\mu \mathrm{m}$-thick) were obtained for immunostaining, terminal deoxynucleotidyl transferase-mediated dUTP nick end labeling (TUNEL) assay and hematoxylin and eosin staining. The study utilized goat anti-cluster of differentiation 34 (CD34) primary antibodies (1:150; R\&D Systems, Minneapolis, MN, USA), a TUNEL detection kit (Roche, San Francisco, CA, USA) and secondary antibodies coupled to biotin (1:200; Proteintech Group, Chicago, IL, USA). Images were captured using an Olympus DP-72 confocal microscope (Olympus Corporation, Tokyo, Japan).

Statistical analysis. Results are expressed as the mean \pm standard error of the mean for three or more independent experiments. To compare data, the analysis of variance test was utilized. A value of $\mathrm{P}<0.05$ was considered to indicate a statistically significant difference.

\section{Results}

Rolipram improves functional outcome and decreases infarction size. To test whether rolipram affected functional outcomes following ischemic stroke, the neurological functional test was performed. Rats treated with rolipram in the 14 days group showed significantly improved functional recovery (based on mNSS testing) compared with the rats in the other groups $(\mathrm{P}<0.05)$ between days 7 and 14 . Rats in the three and seven days groups also showed significantly lower neurological deficits (based on mNSS testing) than the vehicle 

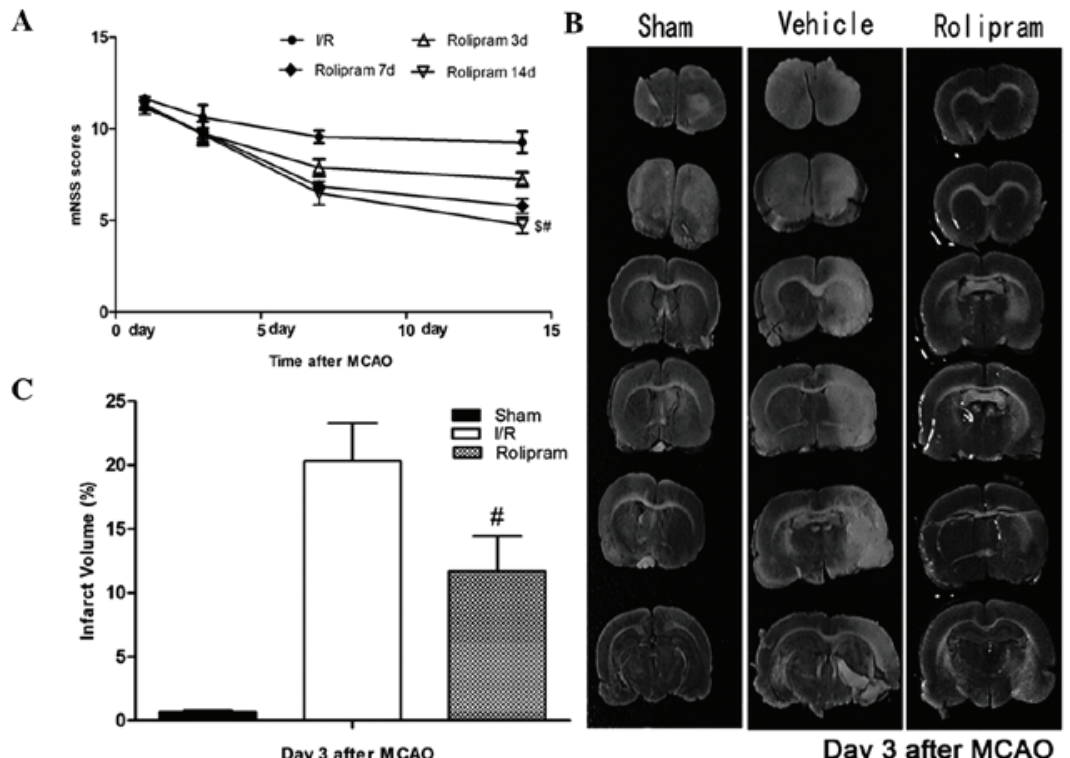

Figure 1. Rolipram improves functional outcome and decreases infarction size. (A) Behavioral tests showed that rolipram significantly improved functional outcome. The mNSSs in groups receiving rolipram therapies were significantly lower than those in the vehicle group $\left({ }^{*} \mathrm{P}<0.01\right)$. In addition, the declining curve of the mNSS in the 14 days group was more evident than that in the seven and three days groups $\left({ }^{\$} \mathrm{P}<0.05\right)$. (B and C) The infarcted area, shown by triphenyltetrazolium chloride staining (white), was prominent in the vehicle group but reduced in the rolipram-treated group $\left({ }^{\#} \mathrm{P}<0.05\right)$. mNSS, modified neurological severity score; SE, standard error.

group on the third, seventh and 14th days after reperfusion; however, the scores in these groups were slightly higher than those in the 14 days group (Fig. 1A). Twenty-four hours after $\mathrm{MCAO}$, the rat brains were evaluated for infarction volume using TTC staining and imaging software (Media Cybernetics, Silver Spring, MD,USA) (19). Representative samples of TTC-stained brain sections are shown in Fig. 1B. Increased areas of white were observed in the brain tissue of the vehicle group compared with the other groups; these areas were associated with increased ischemic injury. The infarcted area shown by TTC staining was decreased in the rolipram-treated groups from $23.4 \pm 1.72$ to $10.34 \pm 2.25 \%$ (Fig. 1C). This indicated that rolipram may attenuate cerebral ischemic injury in rats.

Rolipram increases the p-CREB expression levels in the hippocampus. The ability of rolipram to increase p-CREB levels in the ischemic hemisphere of the ischemic brain was examined by western blot analysis. Ischemic tissues were isolated on the third, seventh and 14th days after reperfusion. Western blotting was performed with specific antibodies. Densitometric quantification of immunoreactive p-CREB (43 kDa) band intensities was performed by normalization to $\beta$-actin, an internal control. As shown in Fig. 2A, $\beta$-actin expression was not different among the groups, and each p-CREB band intensity was therefore corrected to that of $\beta$-actin. Fig. 2B shows that rolipram significantly increased the expression of p-CREB on days 3,7 and 14 in the ischemic hemisphere compared with the sham and vehicle groups $(\mathrm{P}<0.01, \mathrm{n}=5$ per group). A longer duration of rolipram treatment further enhanced the increase in the p-CREB level, and the p-CREB level in the 14 days group was higher than that in the other groups $(\mathrm{P}<0.05)$. These results indicated that rolipram could activate $\mathrm{p}$-CREB in the ischemic brain as well as in cultured neurons.

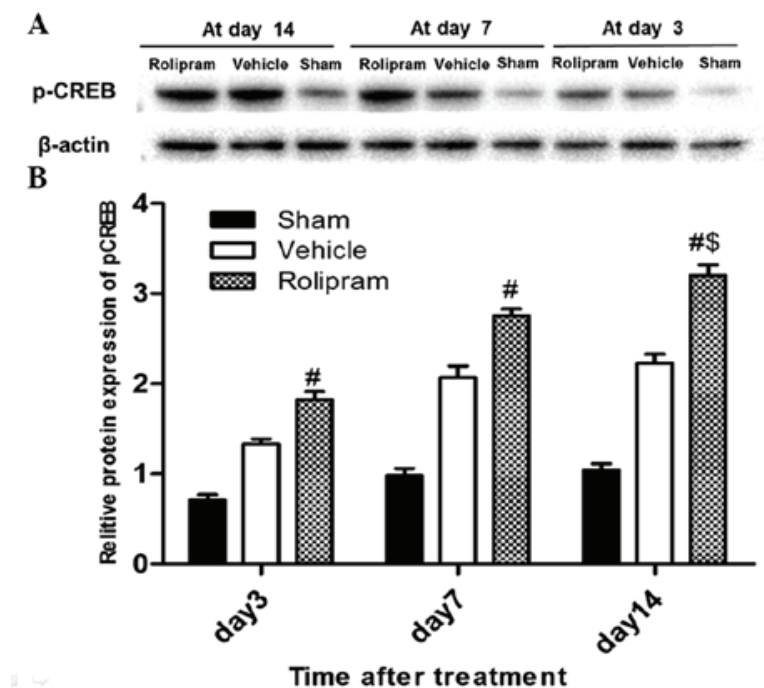

Figure 2. Western blot analysis of p-CREB expression in the ischemic hemisphere. (A) Representative immunoblot images of p-CREB and (B) the relative protein expression of $\mathrm{p}-\mathrm{CREB}$ in the sham, vehicle and rolipram groups on days 3, 7 and 14. Significant differences in p-CREB expression were observed between the rolipram group and the sham and vehicle groups at each time-point ( $\left.{ }^{\#} \mathrm{P}<0.01\right)$, as well as between the groups treated with rolipram for 14 and 7 days $\left({ }^{\$} \mathrm{P}<0.05\right)$ and for 14 and 3 days $\left({ }^{\$} \mathrm{P}<0.05\right)$. Data are presented as the mean \pm standard deviation; $n=5$ per group. $p$-CREB, phosphorylated cyclic adenosine monophosphate-responsive element binding protein.

Rolipram attenuates neuronal apoptosis and stimulates angiogenesis. To examine whether the improved functional outcome induced by rolipram was mediated via a reduction in the number of apoptotic cells, the number of TUNEL-positive cells in the ischemic boundary region was measured. No apoptotic cells 
A
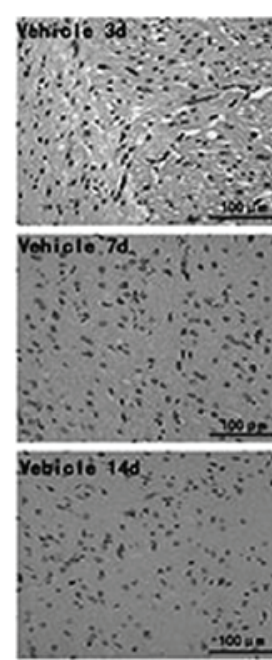
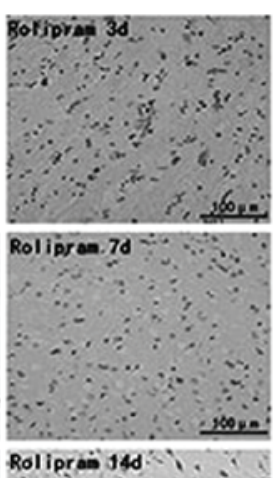

B

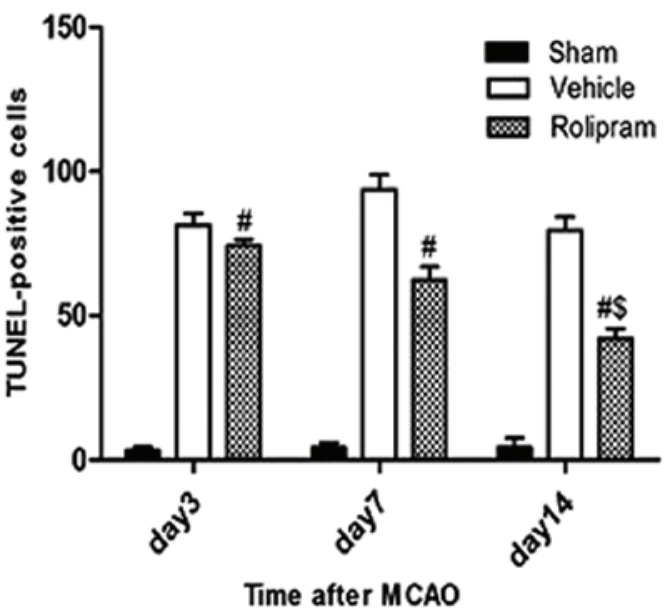

Figure 3. Rolipram attenuates neuronal apoptosis in the ischemic brain. (A) TUNEL-positive cells were frequently observed in the ischemic boundary area. (Vehicle groups, magnification, x200; Rolipram groups, magnification, x400). (B) At day 14 after middle cerebral artery occlusion treatment, the rolipram group exhibited a significantly reduced number of TUNEL-positive cells $\left({ }^{\#} \mathrm{P}<0.01\right)$ compared with the vehicle group. Additionally, the rolipram group treated for 14 days had a significantly reduced number of TUNEL-positive cells compared with the rolipram groups treated for 7 and 3 days, and the vehicle groups $\left({ }^{\$} \mathrm{P}<0.05\right)$. TUNEL, terminal deoxynucleotidyl transferase-mediated dUTP nick end labeling.
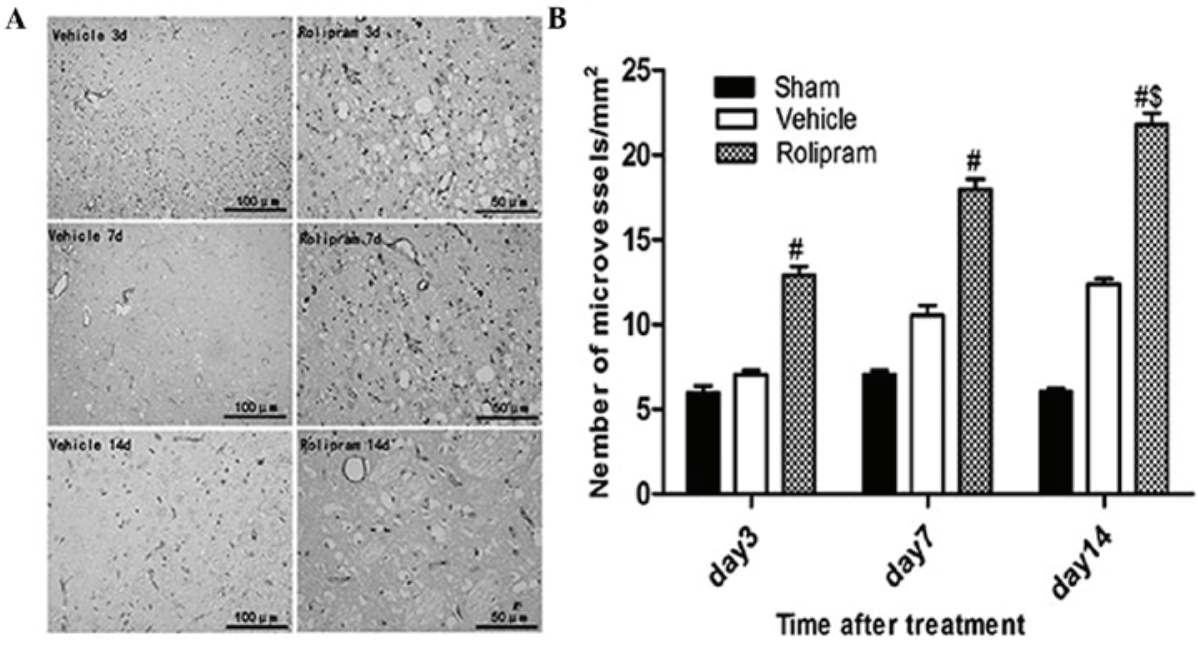

Figure 4. Immunohistochemical staining of $\mathrm{CD} 34^{+}$microvessels in the different treatment groups. (A) $\mathrm{CD} 34^{+}$microvessels in the ischemic hemisphere (magnification, $\mathrm{x} 400$ ). (B) $\mathrm{CD} 34^{+}$microvessels in the sham, vehicle and rolipram-treated groups at different time-points; the rolipram-treated group showed a significantly higher number of $\mathrm{CD} 34^{+}$microvessels than the vehicle and sham groups $\left({ }^{*} \mathrm{P}<0.01\right)$, and the rolipram group with longer treatment duration exhibited a higher number of $\mathrm{CD} 34^{+}$microvessels $\left({ }^{\$} \mathrm{P}<0.05\right)$. CD34, cluster of differentiation 34 .

were detected in the brains from the sham-operated rats (data not shown); however, the rolipram group exhibited a significantly reduced number of TUNEL-positive cells compared with the vehicle group $(\mathrm{P}<0.01$, Fig. 3). Additionally, the 14 days group exhibited a reduced number of TUNEL-positive cells compared with the seven and three days and vehicle groups $(\mathrm{P}<0.05$, Fig. 3). Collectively, these data indicated that rolipram had the capability to attenuate neuronal apoptosis in the ischemic brain.

To determine whether rolipram increased angiogenesis via recruiting $\mathrm{CD} 34^{+}$cells, which differentiate into vascular endothelial cells, in the ischemic boundary region, the number of $\mathrm{CD}_{3} 4^{+}$microvessels $/ \mathrm{mm}^{2}$ in the ischemic hemisphere was analyzed on day 14 after ischemic stroke. The number of $\mathrm{CD}_{34} 4^{+}$microvessels $/ \mathrm{mm}^{2}$ was significantly greater in the rolipram-treated group as compared with that in the vehicle-treated $(21.78 \pm 0.47$ vs. $16.27 \pm 0.32 ; \mathrm{P}<0.05)$ and the sham (21.78 \pm 0.47 vs. $7.23 \pm 0.47$; $\mathrm{P}<0.01)$ groups. Furthermore, in the rolipram group with the longest treatment duration, the number of microvessels in the ischemic boundary region appreciably increased when compared with that in the groups treated for a shorter period of time ( $20.78 \pm 0.47$ vs. $17.96 \pm 0.62$ and $12.93 \pm 0.50$; $\mathrm{P}<0.05$ ) (Fig. 4). These results indicated that rolipram treatment increases the microvessel density (MVD) in the ischemic brain.

\section{Discussion}

Ischemic stroke is currently a significant worldwide health issue and can lead to serious long-term disability. Ischemia can 
stimulate an increase in CREB phosphorylation in neurons (20). CREB belongs to the family of leucine zipper transcription factors that are expressed in a variety of tissues. CREB functions as an effector molecule that initiates changes in the cellular response to extracellular stimuli. Among various gene regulatory factors, CREB has been suggested to be involved in the development and plasticity of neurons, as well as numerous other neuronal processes. p-CREB is the active form of CREB, and CREB phosphorylation at the serine 133 site is believed to be crucial in CREB-dependent transcription. p-CREB regulates cell proliferation, differentiation and survival in the developing brain, and mediates a number of responses, including neuronal plasticity, learning and memory, in the adult brain (21). Using a monkey model of ischemia-enhanced hippocampus neurogenesis, Boneva and Yamashima (20) recently revealed that the expression of p-CREB was significantly upregulated between days 5 and 15 after transient global brain ischemia. In a number of cellular contexts, CREB is transiently activated by its phosphorylation, lasting only 30-60 min (22); however, CREB phosphorylation is persistent in neurons in newborn animals and lasts for as long as 2-3 weeks in rodents $(23,24)$ and $\geq 10$ days in monkeys. Phosphodiesterase-4 (PDE4) inhibitors may also promote p-CREB expression and enhance the survival time of ischemic neurons (5). In the present study, rolipram was administered to rats that had undergone $\mathrm{MCAO}$, with the purpose of exploring whether rolipram could promote angiogenesis and reduce apoptosis following cerebral ischemia, and whether the protective effect was exerted through the CREB signaling pathway.

Phosphorylation of CREB can be caused by a number of upstream signaling cascades, including the cAMP-PKA cascade, the mitogen-activated protein kinase signaling pathway, and the calmodulin-dependent kinases II and IV and phospholipase C-PKC signaling cascades $(25,26)$. Among the above signaling pathways, the CREB phosphorylation that is triggered by the cAMP-PKA cascade has been well studied (27). As described above, the level of p-CREB in the hippocampus increases following hypoxic-ischemic injury; therefore, increasing p-CREB levels may a potential strategy for the treatment of cerebral ischemia. To date, the role of p-CREB in ischemic injury following experimental tMCAO has been explored in diverse pharmacological interventions $(28,29)$. In the present study, rolipram treatment lasting for three, seven and 14 days was utilized. Fig. 2B showed that the administration of rolipram in ischemic rats could induce CREB phosphorylation in the right hemisphere (the ischemic region). Furthermore, the expression level was higher in the 14 days than the three days group, which indicated that rolipram had the ability to enhance the level of p-CREB. The results showed that rolipram administration for a longer period of time induced enhanced protection in the ischemic rats, which was consistent with previous results found in studies using donepezil (28) and resveratrol (29). These previous studies showed that increasing the level of p-CREB could not only ameliorate focal ischemia-induced neuronal death but also the level of the downstream protein B-cell lymphoma 2 (Bcl-2) in the ischemic cortex of rats with tMCAO. It has also been indicated that propofol and ketamine can provide neuroprotection through the inhibition of neuron-specific p-CREB dephosphorylation in the peri-infarct region of mice with permanent MCAO (5). The present study demonstrated that rolipram exerted neuroprotective effects in brain ischemia through the induction of CREB production, which was likely mediated by activation of the cAMP-PKA cascade; an investigation using a CREB inhibitor is now required. Small interfering (si)RNA or a repeated silencer would be necessary for this investigation. Repeated silencer is an intervention measure that is used on animals during the experimental process and can eventually cause CREB in nerve cells to lose its function by blocking its phosphorylation or lowering the expression of CREB protein. A previous study showed that delayed hyperbaric oxygen therapy (HBOT) could decrease the infarct size and cause a neurobehavioral improvement. Furthermore, gene silencing with CREB siRNA or protein phosphatase $1-\gamma$ siRNA attenuated the acute beneficial effects of the HBOT (30). Therefore, we hypothesized that the PDE4 inhibitor rolipram is an antagonistic treatment that could induce angiogenesis and this was one of the experiments in the present study.

PDE4, one of the 11 PDE families (PDE1-11), can hydrolyze cAMP in neuronal tissue, which also plays an important role in the neurochemical and pathological alterations of brain ischemia $(31,32)$. Therefore, we believe that targeting PDE4 may be an innovative approach to treat cognitive disorders associated with cerebral ischemia. Rolipram, as a prototypical PDE4 inhibitor, is widely used in ischemic stroke studies $(15,27)$. A previous study found that rolipram could increase cAMP accumulation; cAMP accumulation activates cAMP-dependent PKA and subsequently phosphorylates and activates CREB (27). Authors in a different study held the view that rolipram could reduce the distracted platform searches induced by cerebral ischemia (15). In the present study, Fig. 1 showed that rolipram could effectively reduce the infarct size and improve neurobehavioral scores, as demonstrated by a lower mNSS and decreased area of TTC staining in the tMCAO model following the use of rolipram for two weeks.

A number of previous studies have demonstrated that CD34 progenitor cells are involved in tissue repair, which can restore the blood perfusion of the ischemic site in ischemic diseases and traumatic injuries by vasculogenesis and angiogenesis $(33,34)$. Compared with the neuron which has ischemic necrosis, the neuron in ischemic penumbra does not have serious metabolic disturbance due to the collateral circulation (35). By creating vascular pathways following ischemia in order to recover the supply of oxygen and sugar as soon as possible is likely to determine whether the neuron can survive or not. This is why the present study focuses on the combination of rolipram's promotion of revascularization and inhibition of apoptosis. Figs. 3 and 4 showed that fewer TUNEL-positive cells and an increased number of $\mathrm{CD}_{3} 4^{+}$microvessels were present in the tissues obtained from rats that were treated with rolipram. This meant that rolipram stimulated angiogenesis and attenuated neuronal apoptosis in areas damaged by ischemia. The MVD counted on day 14 demonstrated the association between neurological and functional recovery and the early improvement in the number of microvessels. In addition, the western blotting data (Fig. 2) were consistent with the immunohistochemistry data (Figs. 3 and 4) in indicating a functional link between angiogenesis/apoptosis resistance and $\mathrm{p}$-CREB.

It is well known that enhancing angiogenesis and reducing the apoptosis of nerve cells may improve brain function in cerebral ischemic mammals. However, the occurrence of 
endogenous neurogenesis following ischemic stroke is early, short-lived and delays neuronal cell death for several days (2). The present results showed that rolipram can attenuate neuronal apoptosis and increase cell proliferation and survival rate in the peri-infarct region through the activation of the CREB pathway, and may therefore be a novel therapeutic strategy to promote brain function recovery following stroke. Subsequent studies should investigate how rolipram enhances angiogenesis, attenuates neuronal apoptosis and affects other relevant transcription factors, including vascular endothelial growth factor, hypoxia inducible factor 1 and $\mathrm{Bcl}-2 / \mathrm{Bcl}-2$-associated $\mathrm{X}$ protein, which are involved in the ischemia-induced angiogenesis (5,36-38).

\section{Acknowledgements}

The authors would like to thank Professor Ying Mao for the guidance provided during the study.

\section{References}

1. Lee YS, Chio CC, Chang CP, et al: Long course hyperbaric oxygen stimulates neurogenesis and attenuates inflammation after ischemic stroke. Mediators Inflamm 2013: 512978, 2013.

2. Okuyama S, Shimada N, Kaji M, et al: Heptamethoxyflavone, a citrus flavonoid, enhances brain-derived neurotrophic factor production and neurogenesis in the hippocampus following cerebral global ischemia in mice. Neurosci Lett 528: 190-195, 2012.

3. Yang J, Shi QD, Song TB, et al: Vasoactive intestinal peptide increases VEGF expression to promote proliferation of brain vascular endothelial cells via the cAMP/PKA pathway after ischemic insult in vitro. Peptides 42: 105-111, 2013.

4. Genovese T1, Mazzon E, Paterniti I, Esposito E and Cuzzocrea S. Neuroprotective effects of olprinone after cerebral ischemia/reperfusion injury in rats. Neurosci Lett 503: 93-99, 2011.

5. Shu L, Li T, Han S, et al: Inhibition of neuron-specific CREB dephosphorylation is involved in propofol and ketamine-induced neuroprotection against cerebral ischemic injuries of mice. Neurochem Res 37: 49-58, 2012.

6. Bourtchuladze R, Frenguelli B, Blendy J, et al: Deficient long-term memory in mice with a targeted mutation of the cAMP-responsive element-binding protein. Cell 79: 59-68, 1994

7. Josselyn SA, Shi C, Carlezon WA Jr, Neve RL, Nestler EJ and Davis M: Long-term memory is facilitated by cAMP response element-binding protein overexpression in the amygdala. J Neurosci 21: 2404-2412, 2001

8. Yamashima T: 'PUFA-GPR40-CREB signaling' hypothesis for the adult primate neurogenesis. Prog Lipid Res 51: 221-231, 2012.

9. Carlezon WA Jr, Duman RS and Nestler EJ: The many faces of CREB. Trends Neurosci 28: 436-445, 2005.

10. Merz K, Herold S and Lie DC: CREB in adult neurogenesis - master and partner in the development of adult-born neurons? Eur J Neurosci 33: 1078-1086, 2011.

11. Wei Z, Belal C, Tu W, et al: Chronic nicotine administration impairs activation of cyclic AMP-response element binding protein and survival of newborn cells in the dentate gyrus. Stem Cells Dev 21: 411-422, 2012.

12. Chava KR, Tauseef M, Sharma T and Mehta D: Cyclic AMP response element-binding protein prevents endothelial permeability increase through transcriptional controlling p190RhoGAP expression. Blood 119: 308-319, 2012.

13. Ma W, Zheng WH, Powell K, Jhamandas K and Quirion R: Chronic morphine exposure increases the phosphorylation of MAP kinases and the transcription factor CREB in dorsal root ganglion neurons: an in vitro and in vivo study. Eur J Neurosci 14: 1091-1104, 2001

14. Li LX, Cheng YF, Lin HB, Wang C, Xu JP and Zhang HT: Prevention of cerebral ischemia-induced memory deficits by inhibition of phosphodiesterase-4 in rats. Metab Brain Dis 26: 37-47, 2011.

15. Li YF, Huang Y, Amsdell SL, Xiao L, O'Donnell JM and Zhang HT: Antidepressant- and anxiolytic-like effects of the phosphodiesterase-4 inhibitor rolipram on behavior depend on cyclic AMP response element binding protein-mediated neurogenesis in the hippocampus. Neuropsychopharmacology 34: 2404-2419, 2009.
16. Li YF, Cheng YF, Huang Y, et al: Phosphodiesterase-4D knock-out and RNA interference-mediated knock-down enhance memory and increase hippocampal neurogenesis via increased cAMP signaling. J Neurosci 31: 172-183, 2011.

17. Chen C, Hu Q, Yan J, et al: Multiple effects of $2 \mathrm{ME} 2$ and D609 on the cortical expression of HIF-1alpha and apoptotic genes in a middle cerebral artery occlusion-induced focal ischemia rat model. J Neurochem 102: 1831-1841, 2007.

18. Sasaki T, Kitagawa K, Omura-Matsuoka E, et al: The phosphodiesterase inhibitor rolipram promotes survival of newborn hippocampal neurons after ischemia. Stroke 38: 1597-1605, 2007.

19. Shu L, Li T, Han S, Ji F, Pan C, Zhang B and Li J: Inhibition of neuron-specific CREB dephosphorylation is involved in propofol and ketamine-induced neuroprotection against cerebral ischemic injuries of mice. Neurochem Res 37: 49-58, 2012.

20. Boneva NB and Yamashima T: New insights into 'GPR40-CREB interaction in adult neurogenesis' specific for primates. Hippocampus 22: 896-905, 2012.

21. Lonze BE and Ginty DD: Function and regulation of CREB family transcription factors in the nervous system. Neuron 35: 605-623, 2002.

22. Ji Y, Lu Y, Yang F, et al: Acute and gradual increases in BDNF concentration elicit distinct signaling and functions in neurons. Nat Neurosci 13: 302-309, 2010.

23. Jagasia R, Steib K, Englberger E, et al: GABA-cAMP response element-binding protein signaling regulates maturation and survival of newly generated neurons in the adult hippocampus. J Neurosci 29: 7966-7977, 2009.

24. Herold S, Jagasia R, Merz K, Wassmer K and Lie DC: CREB signalling regulates early survival, neuronal gene expression and morphological development in adult subventricular zone neurogenesis. Mol Cell Neurosci 46: 79-88, 2011.

25. Johannessen M, Delghandi MP and Moens U: What turns CREB on? Cell Signal 16: 1211-1227, 2004.

26. Nair A and Vaidya VA: Cyclic AMP response element binding protein and brain-derived neurotrophic factor: molecules that modulate our mood? J Biosci 31: 423-434, 2006.

27. Barros DM, Izquierdo LA, Sant'Anna MK, et al: Stimulators of the cAMP cascade reverse amnesia induced by intra-amygdala but not intrahippocampal KN-62 administration. Neurobiol Learn Mem 71: 94-103, 1999

28. Min D, Mao X, Wu K, et al: Donepezil attenuates hippocampal neuronal damage and cognitive deficits after global cerebral ischemia in gerbils. Neurosci Lett 510: 29-33, 2012.

29. Shin JA, Lee KE, Kim HS and Park EM: Acute resveratrol treatment modulates multiple signaling pathways in the ischemic brain. Neurochem Res 37: 2686-2696, 2012.

30. Mu J, Ostrowski RP, Soejima Y, et al: Delayed hyperbaric oxygen therapy induces cell proliferation through stabilization of cAMP responsive element binding protein in the rat model of MCAo-induced ischemic brain injury. Neurobiol Dis 51: 133-143, 2013.

31. Zhang HT: Cyclic AMP-specific phosphodiesterase-4 as a target for the development of antidepressant drugs. Curr Pharm Des 15: 1688-1698, 2009.

32. Zhang HT: Phosphodiesterase targets for cognitive dysfunction and schizophrenia - a New York Academy of Sciences meeting. IDrugs 13: 166-168, 2010.

33. Kao CH, Chen SH, Chio CC and Lin MT: Human umbilical cord blood-derived $\mathrm{CD} 34^{+}$cells may attenuate spinal cord injury by stimulating vascular endothelial and neurotrophic factors. Shock 29: 49-55, 2008.

34. Li S, Wei M, Zhou Z, Wang B, Zhao X and Zhang J: SDF-1 $\alpha$ induces angiogenesis after traumatic brain injury. Brain Res 1444: 76-86, 2012.

35. Krupinski J1, Kaluza J, Kumar P, Kumar S and Wang JM: Role of angiogenesis in patients with cerebral ischemic stroke. Stroke 25: 1794-1798, 1994.

36. Yang J, Shi QD, Song TB, Feng GF, Zang WJ, Zong CH and Chang L: Vasoactive intestinal peptide increases VEGF expression to promote proliferation of brain vascular endothelial cells via the cAMP/PKA pathway after ischemic insult in vitro. Peptides 42: 105-111, 2013.

37. Baugh JA, Gantier M, Li L, Byrne A, Buckley A and Donnelly SC: Dual regulation of macrophage migration inhibitory factor (MIF) expression in hypoxia by CREB and HIF-1. Biochem Biophys Res Commun 347: 895-903, 2006.

38. Leung KW, Ng HM, Tang MK, Wong CC, Wong RN and Wong AS: Ginsenoside-Rg1 mediates a hypoxia-independent upregulation of hypoxia-inducible factor- $1 \alpha$ to promote angiogenesis. Angiogenesis 14: 515-522, 2011. 